\title{
LOS DICCIONARIOS BILINGÜES ESPAÑOL-PORTUGUÉS DESDE EL PRINCIPIO HASTA EL SIGLO XIX
}

Dieter Messner

Universidad de Salzburgo

\author{
RESUMEN \\ Enumeración y descripción de los diccionarios bilingües de las lenguas española y \\ portuguesa. \\ Palabras clave: Diccionarística; español; portugués. \\ ABSTRACT:
}

History of the bilingual dictionaries of Spanish and Portuguese.

Keywords: Lexicography; spanish; portuguese.

Querida Espanha, nunca nos correspondemos muito.

Também, nunca tivemos nada urgente a dizer-nos.

Fernando Venâncio

La lexicografía bilingüe de las lenguas castellana y portuguesa empieza sólo en la segunda mitad del siglo XIX, con la publicación de los tres volúmenes de un diccionario español-portugués, el "primero que se ha publicado, [...] compuesto sobre los mejores diccionarios de las dos naciones", como nos indica el autor, Manoel do Canto e Castro Mascarenhas Valdez ${ }^{1}$, ya en el título.

Hasta finales del siglo XIX siguen algunos diccionarios más: los autores son Jorge César de Figaniere (et al.) ${ }^{2}$, Henrique António Marques ${ }^{3}$, Wildik ${ }^{4}$ e Isidro Monsó ${ }^{5}$. Con

\footnotetext{
${ }^{1}$ Diccionario Español-Portugués, el primero que se ha publicado con las voces, frases, refranes y lucuciones usadas en España y Américas Españolas, en el lenguaje comun antiguo y moderno las ciencias y artes de medicina, veterinaria, quimica, mineralojia, historia natural y botanica comercio y nautica, con algunos nombres propios, y asi las voces particulares de las Provincias Españolas y Americanas, etc. [...] por Manuel do Canto e Castro Mascarenhas Valdez, Hidalgo Caballero de la Casa Real, Lisboa, en la Imprenta Nacional, 1864-66.

${ }^{2}$ Diccionario hespanhol-portuguez e portuguez-hespanhol com phrases e locuções usadas em Hespanha e na America hespanhola, de sciencias e artes, de medicina, chimica, botanica, historia, commercio, marinha, etc. e coordenado dos melhores dicionarios das duas nações. Collaboradores. Conselheiro Jorge Cesar de Figaniere [...]. T. Primeiro, Porto, Empreza Editora de Obras Classicas e Illustradas, 1879. Diccionario portugués-español y español-portugués con frases y locuciones usadas en España y Portugal y sus posesones en Ultramar y las de Ciencias y Artes de medicina, química, botánica, historia, comercio, marina, etc. Colaboradores. Consejero Jorge Cesar de Figaniere [...]. T. Segundo, Porto, Empresa Editora de Obras Clasicas y Ilustradas, 1880.

${ }^{3}$ Novo diccionario hespanhol-portuguêz [...], Lisboa, A. M. Pereira, 1897.
} 
excepción de un importante estudio de Pilar Salas Quesada ${ }^{6}$, no existen análisis pormenorizados de estos diccionarios bilingües ${ }^{7}$. Se constata, pues, que la lengua portuguesa entró bastante tarde en la diccionarística bilingüe del español, al contrario de lo que ocurrió con otras lenguas que se documentan en este mismo número de Philologia Hispalensis. El recién publicado Nuevo Tesoro Lexicográfico del Español (s. XIV-1726) permite conocer todas las obras lexicográficas publicadas antes de 1726.

Tres de los diccionarios son unidireccionales: dos español-portugués (v. notas 1, 3), uno portugués-español (v. nota 5), los otros bidireccionales (v. notas 2, 4). Pilar Salas Quesada ${ }^{9}$ constató que Valdez se había servido para la redacción de su obra del diccionario de la Real Academia Española y había copiado también varias entradas de diccionarios técnicos. Según ella, "muchas de las definiciones en portugués están inspiradas por estos lexicógrafos en las obras monolingües del español". Y concluye afirmando que los diccionarios posteriores español-portugués dependen de una u otra manera de Valdez.

El portugués, sin embargo, tiene una larga tradición de diccionarios bilingües ${ }^{10}$, primero con el latín y viceversa (tradición que empieza ya con Jerónimo Cardoso ${ }^{11}$, en 1562) y después con lenguas modernas, lenguas asiáticas (chino 1580; japonés 1595) ${ }^{12}$, y lenguas amerindias (tupi 1585) ${ }^{13}$ entre las que hay que destacar las obras de los misioneros.

\footnotetext{
${ }^{4}$ Novo diccionario hespanhol-portuguez e portuguez-hespanhol com a pronuncia figurada em ambas as linguas pelo Visconde de Wildik, Paris, Garnier Irmãos, Livreiros-Editores, 1897. Nuevo diccionario portugués-español y español-portugués con la pronunciación figurada en ambas lenguas por el Vizconde de Wildik. Parte Segunda portugués-español. París. Garnier Hermanos, Libreros-Editores, 1899.

${ }^{5}$ Novo diccionario portuguêz-hespanhol [...], Lisboa, A. M. Pereira, 1900.

${ }^{6}$ Agradezco a la autora el haberme facilitado el manuscrito de su estudio inédito sobre la lexicografía con el español y el portugués.

${ }^{7}$ Cf. también: P. Salas Quesada, "Cuatro pequeños vocabularios como testimonio de la lexicografía hispanoportuguesa del siglo XIX”, en prensa.
}

${ }^{8}$ L. Nieto y M. Alvar, Madrid, Arco / Libros, 2007, 11 vols. Cf: "Fuentes por orden cronológico", vol. 1., pp. LXXIX-XCIII.

${ }^{9} \mathrm{~V}$. nota 6.

${ }^{10}$ St. Ettinger, "Die zweisprachige Lexikographie mit Portugiesisch", F.-J. Hausmann et al. (eds.), Wörterbücher, Dictionaries, Dictionnaires, Berlín / Nueva York, De Gruyter, 1991, t. 3, 3020-3030. Una lista de todos los diccionarios portugueses hasta 1804 se encuentra en T. Verdelho y J. P. Silvestre (eds.), Dicionarística Portuguesa. Inventariação e estudo do património lexicográfico, Aveiro, Universidade, 2007, 62-81. Cf. D. Messner, Dicionário dos dicionários portugueses (1551-1858), Salzburgo, Universidad, 1992-2007.

${ }^{11}$ T. Verdelho, "Dicionários de Jerónimo Cardoso", Dicionarística Portuguesa. Inventariação e estudo do património lexicográfico, T. Verdelho y J. P. Silvestre (eds.), Aveiro, Universidade, 2007, 90-114. P. Teyssier, "Jerónimo Cardoso et les origines de la lexicographie portugaise", Bulletin des Études Portugaises et Brésiliennes 41, 1980, 7-32, reed. en Études de Littérature et de Linguistique, París, Fondação C. Gulbenkian, 1990, 199-230.

${ }^{12}$ Lenguas asiáticas: D. Lopes, Expansão da língua portuguesa no Oriente nos séculos XVI, XVII e XVIII, reedição actualizada com notas e prefácio de Luís de Matos, Porto, Portucalense, 1969. D. Messner, "Sobre dicionários portugueses antigos: uma inventariação I", Lusorama 18, 1995, 45-64. Chino: D. Messner, "The First Portuguese Bilingual Dictionary Resorting to a Foreign 'Modern' Language - Chinese", Review of Culture ser. 2 (34-35), January/June 1998, 281-291 (Macau, Instituto Cultural de Macau). Japonés: D. Messner, "Ist das 
En el siglo XVI encontramos obras de diversa finalidad que contienen nomenclaturas ${ }^{14}$ en las que figuran tanto el castellano como el portugués: Lusitanus $1554^{15}$, Laguna $1555^{16}$, que probablemente sea la fuente de un vocabulario de Agustín de $1626^{17}$. También a partir de 1598 los Colloquia $[\ldots]^{18}$ tienen una columna en portugués ${ }^{19}$. En un diccionario plurilingüe de 1617, ya entró asimismo el portugués ${ }^{20}$.

Sólo en 1701 aparece el primer diccionario portugués con una lengua europea, la inglesa (A. J. ${ }^{21}$; el segundo diccionario con inglés es de $1773^{22}$ ), seguida en 1714 por el neerlandés ${ }^{23}$, el francés en $1764^{24}$ y el alemán en $1811^{25}$.

Dictionarium Latino Lusitanicum, ac Iaponicum ein Wörterbuch der portugiesischen Sprache? Zur Rezeption Calepinos in Portugal", Lusorama 28, 1999, 48-52.

${ }^{13}$ M. C. Drumond Mendes Barros / A. L Salim Lessa, "Estudo preliminar de um dicionário português-tupi do período pombalino", Revista de Estudos da Linguagem 3/2, 2005, 73-94 (UFMG, Belo Horizonte).

${ }^{14}$ P. Salas Quesada, "La marca HISP. en los diccionarios plurilingües. En busca de los inicios de la lexicografía hispano-portuguesa", Res Diachronicae Virtual 4 (= El Contacto de Lenguas, $\mathrm{n}^{\circ}$ monográfico coord. por A. Rodríguez y A. García Lenza), 2005, 137-153.

${ }^{15}$ In Dioscoridis Anazarbei de medica materia libros quinque enarrationes eruditissimae Doctoris Amati Lusitani Medici ac Philosophi celeberrimi, quibus non solum Officinarum Seplasiariis, sed bonarum etiam literarum studiosis utilitas adfertur, quum passim simplicia Graece, Latine, Italice, Hispanice, Germanice \& Gallice proponantur, Argentorati: Wendelius Ribelius 1554. Probablemente ya terminado en 1551, según la dedicatoria: "Illustrissimis Viris Rectoribus, et Ampliss. Senatui Rhacusino: Amatus Lusitanus Medicus Physicus Salutem, \& felicitatem P.D. [...] Romae decimoquinto de Maij M. D. LI."

${ }^{16}$ Pedacio Dioscorides Anazarbeo, Acerca de la materia medicinal, y de los venenos mortiferos, Traduzido de la lengua Griega en la vulgar Castellana [...] por el Doctor Andres de Laguna, Medico de Iulio III. Pont. Max. En Anvers, En casa de Iuan Latio. Anno M. D. LV. Lista alfabética de las palabras portuguesas acompañadas de las palabras de las otras lenguas: D. Messner, "Ein Dicionário dos dicionários portugueses", D. Messner / A Schönberger (eds.), Studien zur portugiesischen Lexikologie, Frankfurt am Main, TFM, 1993 (Akten des 2. Gemeinsamen Kolloquiums der Deutschsprachigen Lusitanistik und Katalanistik: Lusitanistischer Teil), 61-174 (espec. 113-135).

${ }^{17}$ M. Agustín, Libro de los secretos de agricultura casa de campo y pastoral, Perpiñán, L. Roure, 1626. Facsímil del "Vocabulario que se sigue en seys Lenguas [...]" y la lista alfabética de todas las palabras portuguesas acompañadas de las palabras de las otras lenguas: D. Messner, "Ein Dicionário dos dicionários portugueses", Studien zur portugiesischen Lexikologie, D. Messner y A. Schönberger (eds.), Frankfurt am Main, TFM, 1993, 61174 (especialmente 85-109).

${ }^{18}$ Colloquia \& Dictionariolum octo linguarum; Latinae, Gallicae, Belgicae, Teutonicae, Hispanicae, Italicae, Anglicae \& Portugallicae. Liber omnibus linguarum studiosis domi ac foris apprimè necessarius. Ex officina Brunonis Schinckelij. Anno 1598. Venduntur Amstrodami in aedibus Cornelij Nicolai.

${ }^{19}$ Lista alfabética de las palabras portuguesas acompañadas de las palabras de las otras lenguas: en D. Messner, "Ein Dicionário dos dicionários portugueses", Studien zur portugiesischen Lexikologie, D. Messner y A. Schönberger (eds.), Frankfurt am Main, TFM, 1993, 61-174 (especialmente 104-109).

${ }^{20}$ J. Minsheu, Ductor in Linguas, The Guide into the Tongues, Londini: John Browne, 1617 (ed. facsimilar e introducción por J. Schäfer, Nueva York, Scholar's Facsimiles \& Reprints Delmar, 1978).

${ }^{21}$ A Compleat Account of the Portugueze Language. Being a Copious Dictionary of English with Portugueze and Portugueze with English [...] By A. J. London, Printed by R. Janemay, 1701. (The Scolar Press Limited, English Linguistics, A Collection of Facsimile Reprints, selected and edited by R. C. Alston, Menston, 1970). 
Algunos estudiosos pretenden que el parentesco de los dos léxicos español y portugués había hecho superflua, durante los siglos XVII y XVIII, la redacción de un diccionario de las dos grandes lenguas iberorrománicas ${ }^{26}$. Este conocimiento mutuo entre el español y el portugués se documenta en textos literarios, por ejemplo, en el Siglo de Oro. Para ilustrar esta situación citaré un fragmento de Tirso de Molina que data de 1635 (El amor médico, acto II, escena III $)^{27}$ :

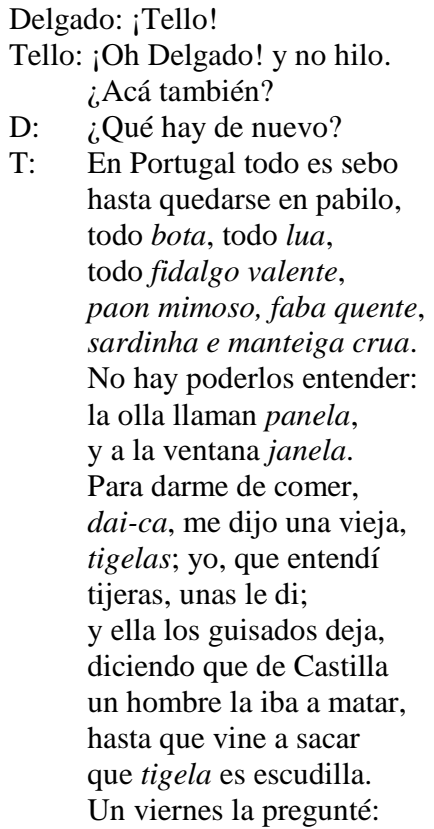

${ }^{22}$ A Dictionary of the Portuguese and English Languages; in two parts, Portuguese and English and English and Portuguese [...] By Anthony Vieyra Transtagano, London, J. Nourse, 1773.

${ }^{23}$ Tesóuro dos Vocábulos Das dûas Línguas Portuguéza, e Bélgica, Woordenschat der twee Taalen Portugeesch, en Nederduitsch, por Mtre. Abrahamo Alewyn, e João Collé, Amsterdamo, pelo Pedro vanden Berge, 1714.

${ }^{24}$ Novo Diccionario das línguas portugueza, e franceza, com os termos latinos, tirado dos melhores Authores, e do Vocabulario Portuguez, e Latino do P. D. Rafael Bluteau [...] pelo Padre Joseph Marques [...] primeira edição; Lisboa, Offic. Patriarcal de Francisco Luiz Ameno, 1764.

${ }^{25}$ Novo Diccionario portuguez-alemão e alemão-portuguez, por João Daniel Wagener; Lipsiae, E. B. Schwickert, 1811.

${ }^{26}$ Por ej., P. Salas Quesada, "La marca HISP. en los diccionarios plurilingües. En busca de los inicios de la lexicografía hispano-portuguesa", Res Diachronicae Virtual 4 (= El Contacto de Lenguas, $\mathrm{n}^{\circ}$ monográfico coord. por A. Rodríguez y A. García Lenza), 2005, 137-153, esp. p. 137.

27 Tirso de Molina, El amor médico, edición de Blanca de los Ríos, Comedias, t. II, Madrid, Aguilar, 1952. Alicante, Biblioteca Virtual Miguel de Cervantes, 1999, 24.4.2008, <http://www.cervantesvirtual.com/ FichaObra.html?Ref=1687> 
«¿Qué tengo de cenar yo?

-Cagados, me respondió.-

Cómalos vuesamercé,

la dije, y pullas a un lado,

que tiene muchas arrugas»;

y supe que eran tortugas

los cagados.

D: ¡Buen guisado!

T: La embajatriz mi señora,

que es digna de todo amor.

y me hace mucho favor,

por no decir me enamora,

da en hablar a lo seboso;

porque en nuestra tierra es fama

que en esta lengua una dama

tiene aire garabatoso;

y entre cosas peregrinas

que suele mandarme hacer,

tracei-me, me dijo ayer,

do jardim umas boninas;

olhai, e un ramo de cravos.

«¿Para qué diablos querrá,

dije, si loca no está,

olla, boñigas y clavos?

El tiempo anda enfermo, y éste

altera nuestra salud;

deben de tener virtud,

sin duda, contra la peste.»

Compré una olla vidriada,

al campo salí, llenéla

de clavos, emboñiguéla,

y llevándola tapada

con la capa, la hallé hablando

con su padre y mi señor

(no era muy fino el olor

con que me iba perfumando.)

Llegué y díjela al oído:

«Aquí aquel recado está»;

y respondióme: «Dai-ca.»

«¿Estás fuera de sentido,

señora, que a esto me obligas?

-repliqué- ¡Gentil humor!

¡Sacarle a un embajador

un puchero de boñigas!»

Mandó que lo descubriese,

y vino a causar su prisa

a unos asco y a otros risa,

y a que mi amo se corriese,

y tuviésemos mohinas.

¡Averigüe Garibay 
que es aquí «mirad»olhai, que las flores son boninas y cravos claveles son! En fin, yo, que su humor sigo, porque se huelgue conmigo, paso plaza de bufón.

En la misma época el jesuita Bento Pereira (1600-1681) publicó en Portugal Prosodia in vocabularium trilingue Latinum, Lusitanicum et Castellanicum digesta [...] (Lisboa, Manuel de Carvalho, 1634), una obra que, a partir de la segunda edición de 1647, fue acompañada por el Thesouro da Lingoa Portuguesa ${ }^{28}$. Pilar Salas Quesada constató ${ }^{29}$ que "La primera edición de la Prosodia comprende cerca de 50000 voces latinas, con su equivalente siempre en portugués, mientras que el español hace aparición únicamente en unas 4500 palabras, un $10 \%$ de las entradas, proporción que se fue ampliando en las siguientes ediciones". A partir de la séptima edición, de 1697, ya no se incluye el español. Es de suponer que su autor pensase, con la inclusión de algunas expresiones españolas, facilitar a hispano-hablantes el acceso a la lengua latina.

Otro testimonio del conocimiento de la lengua española en Portugal, por lo menos dentro de la clase culta, es un libro de 1671 (Joam Franco Barretto, Ortografia da linga portugueza, Em Lisboa, na Officina de Ioam da Costa). Para construir correctamente el plural de algunos sustantivos portugueses el autor aconseja a sus lectores recurrir a las formas correspondientes de la lengua española. Cito este texto según el Diccionario da Lingoa Portugueza, publicado por la Academia Real das Sciencias de Lisboa (Lisboa, Oficina da mesma Academia, 1793, p. 327 ):

ÃO. Diphthongo da lingoa Portugueza, em que acabão muitas palavras. Esta orthographia tem sido disputada por Antigos e Modernos. [...]

Barret. Orthogr. 23 Este diphthongo [ão] succedeo em lugar da antiga terminação dos Portuguezes om, que punhão em lugar de an ou on, dos Castelhanos [...] He o mais frequente de nossa lingoa, e sobre que ha varias opiniões, a minha he, que para o acertarmos, nos guiaremos pela lingoa Castelhana, e os vocabulos, que elles acabarem em ano, anos, acabaremos nós em ão, ãos [...] E assi se escreverão por este diphthongo ão todas as terceiras pessoas do plural, e tempo futuro de qualquer verbo, que seja, como serão, amarão, lerão, ensinarão, forão, \&c.

Son pocos los estudiosos que se han ocupado de un así llamado "Diccionario Castellano y Portuguez para facilitar a los castellanos el uso del Vocabulario Portuguez, y Latino”, que se encuentra insertado en el volumen VIII (con paginación independiente del resto del libro: pp. 25-189) del más voluminoso diccionario de la lengua portuguesa a principios del

\footnotetext{
${ }^{28}$ [...] composto pelo Padre D. Bento Pereyra da Companhia de IESU, Portugues Borbano: Lente que foy da primeira classe de Rhetorica em a Universidade de Evora \& hoje o he da sagrada Theologia em a mesma Universidade e tem todos os vocabulos portugueses que trazem Cardoso \& Barbosa \& de novo outros muytos mil em tanta copia que so os vocabulos accrescentados saõ outros tantos, \& mais, que todos quantos tem os sobreditos Vocabularios [...] Lisboa, na officina de Paulo Craesbaeck, 1647.

29 "El español en la Prosodia in Vocabularium Trilingue (1634) de Bento Pereira", Actas del VI Congreso Internacional de Historia de la Lengua Española, Madrid, Arco / Libros, 2004, 1683-1693, esp. p. 1684.
} 
siglo XVIII, el Vocabulario Portuguez e Latino, [...] autorizado com exemplos dos melhores escritores portuguezes, e latinos, e offerecido a ElRey de Portugal, D. Joaõ V pelo Padre D. Rafael Bluteau [... $]^{30}$. No sabemos cuáles fueron los motivos para incluir muchos topónimos que no se distinguen por su grafía:

Ejemplos:

$\begin{array}{ll}\text { [Esp.] } & \text { [Portug.] } \\ \text { Aa. Nombres de algunos rios } & \text { Idem. } \\ \text { Aade. Ciudad. } & \text { Idem. } \\ \text { Aalem Rio, } & \text { Idem. } \\ \text { Aarac. Ciudad. } & \text { Idem. } \\ \text { Aarasio, Ciudad. } & \text { Idem. } \\ \text { Aaarburgo, Ciudad, } & \text { Idem. }\end{array}$

Otras entradas tampoco difieren entre las dos lenguas, lo que Bluteau documenta así (vol. VIII. p. 15): “[...] las palabras Portuguesas totalmente semejantes a las Castellanas [...] son tantas, y tan comunes a las dos naciones, que [...] para dicho curioso serà quasi escusado dicho Diccionario".

Ejemplos:

[Esp.] [Portug.]

Abdicar, Idem.

Abdomen, Termino Anatomico. Idem.

Abecedario. Id.

Más útil es la "Tabla de palabras portuguezas remotas de la lengua castellana" incluida en el mismo volumen (pp. 15-24) ${ }^{31}$. Bluteau justifica este vocabulario con varios argumentos, de los que el más importante, en mi opinión, es:

[...] Dividan-se los Vocablos Portuguezes en tres classes; una de Vocablos, que con el castellano tienen mucha semejança; otra de Vocablos, totalmente semejantes; y otra de los que tienen poca, ó ninguna analogia. [...] La tercera classe es de las palabras Portuguezas, mas remotas de la lengua Castellana. Destas se allarà una tabla al fin del Diccionario castellano, y Portuguez; conosco, y confiesso que es diminuta, y que havrà muchos otros Vocablos differentes de los Castellanos; pero la mayor parte de los que faltan, son de

\footnotetext{
${ }^{30}$ Coimbra, Collegio das Artes da Companhia de Jesus; Lisboa; na officina de Pascoal da Sylva. T. 1 (A), 1712 ; t. 2 (B-C), 1712; t. 3 (D-E), 1713; t. 4 (F-G-H-I), 1713; t. 5 (K-L-M-N), 1716; t. 6 (O-P), 1720; t. 7 (Q-R-S), 1720; t. 8 (T-U-V-X-Y-Z), 1721. Supplemento ao vocabulario 1, 1727. Supplemento ao vocabulario, Parte segunda, 1728.

${ }^{31}$ Edición: R. Ponce de León / S. Duarte, "O contributo da obra lexicográfica de Rafael Bluteau para a História do ensino do Português como língua estrangeira: O Methodo breve, y facil para entender Castellanos la lengua portugueza", Revista da Faculdade de Letras, Porto, II Série, vol. XXII, 2005, pp. 373-429.
} 
oficinas Fabriles, y materias vulgares, que en estilo culto, y politico rara vezes tienen lugar. (P. 15)

Ejemplos:

[Port.]

Aba de chapêo,

Abada,

Abâno.

Fazer de Abôbada.

Abrandar.

Abûtre. Ave.

Acalentar o menino.

Acarar.

Acinte.

Fazer acintes.

Acotovelar,

Açougue.

Açúde.

Acugular.

Açular o caõ.

Adoçar.

Aduelas.

Affeiçoar alguma cousa. Facionar.

Afferrolhar.

Afinarse de fóme.

Aformosear.
[Esp.]

Ala de sombrero

Regazada.

Aventadèro.

Bobedar.

Calmar.

Buitre.

Arrullar el niño.

Confrontar.

Provocacion Despecho.

Provocar.

Codear. Dar de codo.

Carniceria.

Acequia.

Colmar.

Azuzar.

Adulzar.

Costillas de cubo.

Dar forma.

Ahorrojar.

Ailarse de ambre.

Ermosear.

Como resultado de un análisis detallado de los dos vocabularios de Bluteau, Rogélio Ponce de León dice: “[...] o fim último, quer do Diccionario, quer do Methodo, é, portanto, o conhecimento do latim e não o do Português",32.

El Vocabulario Portuguez e Latino [...], de Bluteau, fue conocido por los lexicógrafos de la Real Academia Española, que mencionan, en el Prólogo del llamado Diccionario de Autoridades $^{33}$, diccionarios extranjeros:

\footnotetext{
32 "O contributo da obra lexicográfica de Rafael Bluteau para a história do ensino do português como língua estrangeira", Revista da Faculdade de Letras - Linguas e Literaturas, Porto, II Série, vol. XXII, 2005, 373-429, espec. p. 378.

${ }^{33}$ Diccionario de la lengua castellana, en que se explica el verdadero sentido de las voces, su naturaleza y calidad, con las phrases o modos de hablar, los proverbios o refranes, y otras cosas convenientes al uso de la lengua [...] Compuesto por la Real Academia Española. Tomo primero. Que contiene las letras A.B. Madrid, Imprenta de Francisco del Hierro, 1726. Tomo segundo. Que contiene la letra C. Madrid, Imprenta de Francisco del Hierro, 1729. Tomo tercero. Que contiene las letras D.E.F. Madrid, Imprenta de la Real Academia Española por la viuda de Francisco del Hierro, 1732. Tomo quarto. Que contiene las letras G.H.I.J.K.L.M.N. Madrid, Imprenta de la Real Academia Española por los herederos de Francisco del Hierro, 1734. Tomo quinto. Que contiene las letras O.P.Q.R. Madrid, Imprenta de la Real Academia Española, por los herederos de Francisco del Hierro, 1737. Tomo sexto. Que contiene las letras S.T.V.X.Y.Z. Madrid, Imprenta de la Real Academia Española, por los herederos de Francisco del Hierro, 1739. Reprod. facs. de la ed. de Madrid, Imp. de Francisco del Hierro, 1726-1737). 3 vols. [1]. A-C [2]. D-Ñ [3]. O-Z, Madrid, Gredos, 1976 (Biblioteca románica hispánica. V, Diccionarios 3).
} 
Los Franceses, Italianos, Ingleses y Portugueses han enriquecido sus Patrias, e Idiomas con perfectissimos Diccionarios (vol. 1, p. XI) ${ }^{34}$.

Y citan a Bluteau a partir del volumen 4, de 1734 (p. 7). Un ejemplo:

Galardon: El premio, recompensa ò retribucion de los méritos, servicios y beneficios. Bluteau en su Diccionario Portugués siente que esta voz se deriva del Francés Guerdón, que antiguamente se decía Guerredon, esto es don o premio de guerra.

La traducción parcial de las obras del médico portugués Curvo Semedo (fallecido en 1719), impresa probablemente en 1731 con el título de Secretos medicos y chirurgicos del Doctor Juan Curbo Semmedo, traducidos de lengua vulgar portuguesa en castellana [...] por D. Thomás Cortijo Herraiz (Madrid, Bernardo Peralta, s. f.), viene acompañada de un pequeño vocabulario (pp. 133-137). En el "Prologo a el lector", el traductor español lo justifica así:

Despues de las Notas pongo un breve Diccionario de los nombres que Curbo usa en sus Obras, que parece se ocultan à la comun inteligencia, por la ninguna conexion que algunas voces tienen con nuestra Lengua Castellana, ni latina: y omito otras muchissimas por la opuesta razon ${ }^{35}$.

El primer diccionario de la Academia Portuguesa ${ }^{36}$, de 1793, antes citado en el contexto de la formación correcta del plural de palabras portuguesas, es también un excelente ejemplo para descubrir la influencia de la lexicografía española sobre la diccionarística portuguesa, sin provocar la necesidad de un diccionario bilingüe de las dos lenguas. El diccionario portugués contiene varias alusiones directas al diccionario de la Academia Española, de 1780, como se ve en el ejemplo siguiente (p. 210):

Alfoz s.m. antiq. Termo ou destricto determinado de terras, herdades, \&c. He voz Arabiga composta do articulo al, e do nome fohosz, que significa veiga ou campo plano, segundo o P. Alcalá. Diccion. Castelh. Leão, Chr. De D. Din. 118 Que o dito D. Affonso de Lacerda houvesse para si [...] Algava e os Montes de Greda, de Magam, a Povoa da Sarria com seu alfoz.

La entrada correspondiente en el Diccionario de la Lengua Castellana $^{37}$ de 1780, p. 49 es:

Alfoz, s.m. ant. Término, ó pago, que se contiene en algun distrito. Es voz árabe compuesta del artículo al, y del nombre fohoz, que significa vega, ó campo llano, según el P. Alcalá. Limes, pagus.

\footnotetext{
${ }^{34}$ B. Gómez-Pablos, "Rafael Bluteau en el Diccionario de Autoridades", Revista de Filología, Universidad de La Laguna, 22, 2004, 67-78.

${ }^{35}$ D. Messner, "Un breve diccionario lusitanico castellano de 1731", Lusorama 47-48, 2001, 122-127.

${ }^{36}$ Dicionário da Língua Portuguesa publicado pela Academia das Ciências de Lisboa M.DCC. XCIII. tomo primeiro A, Reprodução fac-similada assinalando o II Centenário da edição. Lisboa, Academia, 1993. [= DLP].

${ }^{37}$ Diccionario de la lengua castellana reducido a un tomo para su más fácil uso. Madrid, Joaquim Ibarra. Facsímil de la primera edición (1780), Introducción de M. Seco, Madrid, RAE, 1991. [=DRAE].
} 
Pero la influencia que ejerció el diccionario español sobre su homólogo portugués se ve no sólo en las entradas en las que se cita el origen sino también de manera sorprendente e inesperada en muchos otros lemas portugueses. De los ejemplos que he escogido al azar palabras que empiezan con al- sólo reproduzco unos pocos que me parecen lo suficientemente representativos para todos:

DRAE 1780, 36:

Ala. Mil. La parte de tropa que cubre el centro del exército por qualquiera de los dos costados.

DLP 1793, 178:

Ala Milic. A parte da tropa, que cobre o centro do exercito por qualquer dos dous costados.

DRAE 1780, 44:

Alcanzadura. s.f. Alb. La hinchazon que suele hacerse á las caballerias de algun golpe de hierro, ó piedra en la parte trasera del pie junto á la uña, ó casco. Llámase tambien así la contusion, ó herida que se les hace topándose el pie con la mano. [...]

DLP 1793, 190:

Alcançadura. s.f. Alveit. Inchação, que se costuma fazer nas cavalgaduras por algum golpe de ferro o de pedra na parte posterior do pé junto á unha ou casco; ou tambem a contusão ou ferida, que ellas mesmas se fazem a si, tocandose com a ferradura do pé no mão, ou as mãos, huma com outra, quando, correndo ou voltando, se alcanção. [...]

Resumiendo lo antes descrito podemos repetir que la lexicografía bilingüe de las lenguas española y portuguesa surge muy tarde en comparación con otras diccionarísticas ${ }^{38}$. También es verdad que ninguno de los diccionarios aquí citados se imprimió en España ${ }^{39}$.

\section{Dieter Messner}

Dieter.Messner@sbg.ac.at

FB Romanistik

Universität Salzburg

Akademiestrasse 24

5020 Salzburg

\footnotetext{
${ }^{38}$ Una lista de los diccionarios bilingües hasta 2006: D. Messner, "La lexicografía bilingüe portugués-español”, íd., Miscelánea lexicológica iberorrománica, Salamanca, Luso-española de Ediciones, 2008, 137-154.

${ }^{39}$ No quisiera omitir los diccionarios gallegos: Diccionario de diccionarios [recurso electrónico] edición a cargo de A. Santamarina. [Versión 3] [A Coruña] Fundación Pedro Barrié de la Maza, 2003. 1 disco CD-ROM + 1 Guía. (Biblioteca Filolóxica Galega). El primer vocabulario es de 1536: Bachiller Olea, Vocabulos Gallegos escuros lo que quieren dezir, edición y estudio J. L. Pensado, revisado, completado y dispuesto para la imprenta por D. Messner, Real Academia Galega, Cadernos de Lingua, Anexo 7, A Coruña, 2003.
} 